\title{
Endoscopic treatment of bleeding gastric varices with histoacryl (N-butyl-2-cyanoacrylate): a South European single center experience
}

\author{
Pedro Monsanto • Nuno Almeida • Albano Rosa • \\ Fernanda Maçôas • Clotilde Lérias • Francisco Portela • \\ Pedro Amaro • Manuela Ferreira • Hermano Gouveia • \\ Carlos Sofia
}

Received: 12 January 2012 / Accepted: 19 May 2012 /Published online: 6 July 2012

(C) Indian Society of Gastroenterology 2012

\begin{abstract}
Background Endoscopic injection of N-butyl-2-cyanoacrylate is the current recommended treatment for gastric variceal bleeding. Despite the extensive worldwide use, there are still differences related to the technique, safety, and long term-results. We retrospectively evaluated the efficacy and safety of cyanoacrylate in patients with gastric variceal bleeding.

Patients and Methods Between January 1998 and January 2010, 97 patients with gastric variceal bleeding underwent endoscopic treatment with a mixture of N-butyl-2-cyanoacrylate and Lipiodol ${ }^{\mathrm{TM}}$. Ninety-one patients had cirrhosis and 6 had non-cirrhotic portal hypertension. Child-Pugh score at presentation for cirrhotic patients was A-12.1\%; B-53.8 \%; C-34.1\% and median MELD score at admission was 13 (326). Successful hemostasis, rebleeding rate and complications were reviewed. Median time of follow up was 19 months $(0.5-126)$.

Results A median mixture volume of $1.5 \mathrm{~mL}$ ( 0.6 to $5 \mathrm{~mL}$ ), in 1 to 8 injections, was used, with immediate hemostasis rate of $95.9 \%$ and early rebleeding rate of $14.4 \%$. One or more complications occurred in $17.5 \%$ and were associated with the use of Sengstaken-Blakemore tube before cyanoacrylate and very early rebleeding $(p<0.05)$. Hospital mortality rate during initial bleeding episode was $9.3 \%$. Very
\end{abstract}

P. Monsanto $(\bowtie) \cdot$ N. Almeida $\cdot$ A. Rosa $\cdot$ F. Maçôas $\cdot$ C. Lérias $\cdot$

F. Portela $\cdot$ P. Amaro $\cdot$ M. Ferreira $\cdot$ H. Gouveia $\cdot$ C. Sofia

Gastroenterology Department, Coimbra University Hospital,

Praceta Mota Pinto,

3000-075 Coimbra, Portugal

e-mail: pedromonsanto@gmail.com early rebleeding was a strong and independent predictor for in-hospital mortality $(p<0.001)$. Long-term mortality rate was $58.8 \%$, in most of the cases secondary to hepatic failure.

Conclusion N-butyl-2-cyanoacrylate is a rapid, easy and highly effective modality for immediate hemostasis of gastric variceal bleeding with an acceptable rebleeding rate. Patients with very early rebleeding are at higher risk of death.

Keywords Cirrhosis · Hemostasis · Portal hypertension · Variceal bleeding

\section{Introduction}

Over the past two decades, in-hospital mortality of patients with cirrhosis and variceal bleeding decreased threefold as a result of new treatment modalities, combined use of pharmacological and endoscopic therapies, and short-term antibiotic prophylaxis. However, the mortality from a first variceal bleed remains as high as $14.5 \%$ [1]. Although less common than esophageal varices, gastric varices occur in approximately $20 \%$ of patients with portal hypertension. Bleeding from gastric varices is usually more severe and difficult to control and has a higher mortality than esophageal variceal bleeding. After control of acute bleeding, the rebleeding rate can be high, depending on the applied endoscopic therapeutic modality. Soehendra et al. first reported in 1986 that bleeding from gastric varices could be controlled by intravariceal injection of the tissue adhesive agent butyl cyanoacrylate [2]. Since then, several authors 
have used different sclerosing agents to achieve hemostasis, but N-butyl-2-cyanoacrylate (Histoacryl ${ }^{\mathrm{TM}}$ ) is the only promising agent, achieving primary hemostasis in $70 \%$ to $100 \%$ of patients, with an early rebleeding rate ranging from $0 \%$ to $23 \%$ [3-10]. In fact, although sclerotherapy with $5 \%$ ethanolamine oleate, absolute ethanol or $1 \%$ polidocanol has been reported to control bleeding in about $66 \%$ of patients, it has a rebleeding rate of $50 \%$ and it is more effective in patients with gastroesophageal varices than with fundal varices [11]. When compared to absolute ethanol, in a small randomized-controlled trial of patients with isolated fundal varices, cyanoacrylate was significantly more effective in achieving variceal obliteration than alcohol $(100 \%$ vs. $44 \%)$ [12]. Absolute ethanol is also associated with high incidence of complications, in particular, gastric ulcerations. Variceal band ligation is currently the usual therapy for esophageal varices and can also be used with success for gastroesophageal varices, however with lower initial hemostatic rate and higher rebleeding rates than cyanoacrylate [10]. Human thrombin has also been employed for endoscopic variceal obturation in small studies, with reported initial hemostasis rate of $92 \%$ and no rebleeding [13]. Although there is no consensus for the optimal treatment of gastric varices, N-butyl-2-cyanoacrylate tissue glue injection is the only treatment that has been shown to be effective and its use is recommended by Baveno V consensus workshop [14]. There are still differences related to the technique, safety and long term results. Our study was conducted to address the safety and the results with N-butyl-2-cyanoacrylate injection in acute gastric bleeding varices.

\section{Patients and Methods}

Patients Between January 1998 and January 2010, 97 patients were admitted to our facility with acute bleeding from gastric varices and underwent endoscopic injection of N-butyl-2-cyanoacrylate (Histoacryl ${ }^{\mathrm{TM}}$; B. Braun, Melsungen, Germany) combined with Lipiodol ${ }^{\mathrm{TM}}$ (Laboratoire Guerbert, Aulnay-Sous-Bois, France) for hemostasis. The mean $( \pm \mathrm{SD})$ age was $59.6 \pm 11.8$ years with male patients accounting for $80 \%(n=78)$. Liver cirrhosis was the etiology of portal hypertension in 91 patients $(93.8 \%)$ and only 6 patients $(6.2 \%)$ had non-cirrhotic portal hypertension. The main etiology of bleeding gastric varices was alcohol related-cirrhosis in $73(75.3 \%)$, followed by virus in 10 $(10.3 \%)$, alcohol and virus in $4(4.1 \%)$ and other causes in $4(4.1 \%)$. In terms of liver cirrhosis disease severity, 11 patients $(12.1 \%)$ belonged to Child-Pugh A, $49(53.8 \%)$ to Child-Pugh B, and 31 (34.1\%) to Child-Pugh C. Median MELD score at admission was 13 (range: 3 to 26). Mean ( $\pm \mathrm{SD}$ ) hemoglobin level was $8.3 \pm 2.1 \mathrm{~g} / \mathrm{dL}$ and mean prothrombin rate was $56.1 \pm 15 \%$. Clinical presentation was with hematemesis $(48.5 \%)$, hematemesis and melena $(41.2 \%)$ or melena $(10.3 \%)$. Among these patients, 20 (20.6\%) had active bleeding (spurting or oozing) and 77 (79.4\%) high-risk stigmata of recent variceal bleeding, including red wale spots, adherent clot or a plug (nipple) sign on a gastric varix in a patient with recent upper GI bleeding without other potential source of hemorrhage. Some patients were transferred from outside hospitals. Sclerosants were previously unsuccessfully used in 23 patients $(23.7 \%)$ and Sengstaken-Blakemore tube was used for temporary control of bleeding in $25(25.8 \%)$. Clinical characteristics of patients with gastric variceal bleeding are expressed in Table 1.

The location of gastric varices was determined according to the classification described by Sarin and divided into gastroesophageal varices type 1 (GOV1) or gastric varices continuing as an extension of esophageal varices along the lesser curve of the stomach, gastroesophageal varices type 2 (GOV2) or gastric varices continuing as an extension of esophageal varices toward the greater curvature, isolated gastric varices type 1 (IGV1) or fundal varices within a

Table 1 Clinical characteristics of patients with gastric varices

\begin{tabular}{ll}
\hline Characteristics & $N(\%) ;$ mean \pm SD/median (range) \\
\hline Patients & 97 \\
Male:Female & $78: 19$ \\
Age (years) & $59.6 \pm 11.8$ \\
Etiology of gastric varices & \\
Cirrhosis & $91(93.8 \%)$ \\
Alcoholism & $73(75.3 \%)$ \\
Virus & $10(10.3 \%)$ \\
Alcoholism and virus & $4(4.1 \%)$ \\
Others & $4(4.1 \%)$ \\
Non-cirrhotic PHT & $6(6.2 \%)$ \\
Bleeding status & \\
Active bleeding & $20(20.6 \%)$ \\
Recent bleeding & $77(79.4 \%)$ \\
Child-Pugh classification & \\
A & $11(12.1 \%)$ \\
B & $49(53.8 \%)$ \\
C & $31(34.1 \%)$ \\
MELD score & $13(3-26)$ \\
Gastric varices classification & $36(37.2 \%)$ \\
GOV1 & $27(27.8 \%)$ \\
GOV2 & $30(30.9 \%)$ \\
IGV1 & $4(4.1 \%)$ \\
\hline
\end{tabular}

$P T H$ portal hypertension; $G O V$ gastroesophageal varices; $I G V$ isolated gastric varices; $M E L D$ model for end-stage liver disease 
few centimeters of the gastric cardia, and isolated gastric varices type 2 (IGV2) or ectopic varices located in the antrum, corpus and first duodenal portion.

The study is in accordance with the ethical standards of institutional ethics committee and with the Helsinki Declaration. Informed consent was obtained from all patients and/ or their relatives for all procedures.

Methods N-butyl-2-cyanoacrylate was mixed with Lipiodol in a $1: 1$ or $1: 1.5$ ratio. Before injection, the dead space volume of the injection catheter was filled with distilled water. The Histoacryl-Lipiodol mixture was injected directly into gastric varices, followed immediately by an injection of distilled water in the same volume than the dead space to deliver the entire glue from the catheter into the varix. The needle was then retracted and washed with distilled water to flush out any Histoacryl retained in the catheter. The injected volume of the Histoacryl-Lipiodol mixture was based on the variceal dimension and appearance. Reinjection was performed during the same session for persistent bleeding or incomplete obliteration (soft sensation on palpation with the retracted needle) or during a follow up session for poor variceal obliteration and contiguous unobliterated varices. The injection was strictly intravariceal to avoid ulceration. An X-ray film was taken after each session to check the presence and contour of the cyanoacrylate cast or to detect any embolic material in the chest in patients whom were suspected to have cyanoacrylate embolism. All patients received intravenous broad spectrum antibiotics prophylactically and octreotide infusion $(50 \mathrm{mcg} / \mathrm{h}$ for the first $48 \mathrm{~h}$ and $25 \mathrm{mcg} / \mathrm{h}$ in the next $72 \mathrm{~h}$ ) or terlipressin at admission and continued for 5 days. The SengstakenBlakemore tube was used, when necessary, for temporary control of bleeding until the application of the HistoacrylLipiodol mixture, and when hemostasis was not achieved and patients were directed to other therapies.

Outcome measures Initial hemostasis was defined by the presence of stable vital signs and the absence of bleeding within $48 \mathrm{~h}$ after treatment. Rebleeding was defined either as active bleeding from treated varices seen during endoscopy or occurrence of hematemesis and/or melena associated with a decrease in hemoglobin of $>2 \mathrm{~g} / \mathrm{dL}$ or associated with hemodynamic instability. Very early rebleeding was defined as occurring within 48 to $120 \mathrm{~h}$ of initial hemostasis and early rebleeding was defined as occurring after $120 \mathrm{~h}$ and earlier than 6 weeks of initial hemostasis. The outcome measures were immediate hemostasis rate, very early and early rebleeding rate, the bleeding mortality rate and procedure-related complications. Complications included fever, lung effusion, pulmonary embolism, cerebral infarction, septicemia and aspiration pneumonia.
Data analysis Statistical interpretation of data was performed using Statistical Package for Social Sciences version 16.0 for Windows (SPSS, Inc., Chicago, Illinois, USA). Data were expressed as percent, mean \pm standard deviation, or median and range as appropriate. Student $t$ test, $\chi^{2}$ test and Fisher's exact test were used wherever appropriate to assess differences between groups with respect to the mortality and complication rates $(p<0.05$ was considered statistically significant). With the significant prognostic variables multivariate logistic regression was carried out using forward stepwise (conditional) method to select independent predictors of mortality.

\section{Results}

A total of 97 patients with bleeding gastric varices underwent endoscopic obliteration with N-butyl-2-cyanoacrylate injection from January 1998 to January 2010. The mean transfusion requirement for packed red blood cells during the index bleeding episode was $4.7 \pm 3.6$ units. According to Sarin's classification of gastric varices, GOV1 were detected in 36 patients $(37.2 \%)$, GOV2 in $27(27.8 \%)$, IGV1 in 30 $(30.9 \%)$ and IGV2 in 4 (4.1\%) (Table 1$)$. Median time from admission until first cyanoacrylate injection was $24 \mathrm{~h}$ (range: 3 to 360 ), and a median mixture volume of $1.5 \mathrm{~mL}$ (range: 0.6 to $5 \mathrm{~mL}$ ) was injected, in 1 to 8 injections per procedure (median 2).

Treatment efficacy The overall success rate for achieving initial hemostasis with N-butyl-2-cyanoacrylate was $95.9 \%$, without recurrent bleeding within $48 \mathrm{~h}$. Rebleeding occurred in $14(14.4 \%)$ patients after $48 \mathrm{~h}$ : very early rebleeding in 3 patients $(3.1 \%)$ and early rebleeding in 11 patients $(11.3 \%)$. Primary hemostasis failure and rebleeding were statistically independent of hepatic function and sub-types of gastric varices. However there were no rebleeding or primary hemostasis failure in IGV2 and non-cirrhotic portal hypertension. Secondary hemostasis with cyanoacrylate was applied in $8(44.4 \%)$ patients with no initial hemostasis or with rebleeding.

In-hospital mortality rate was $9.3 \%$. Factors associated with in-hospital mortality $(p<0.05)$ were: poor hepatic function (Child-Pugh C), no immediate hemostasis with cyanoacrylate, need for Sengstaken-Blakemore tube after cyanoacrylate, presence of complications, very early rebleeding and prothrombin rate inferior to $42 \%$. Multivariate logistic regression with the significant prognostic variables (Child-Pugh class was excluded from analysis as some patients had a non-cirrhotic portal hypertension; need for Sengstaken-Blakemore tube after cyanoacrylate was also excluded because these patients were the same as those that 
did not achieve immediate hemostasis with cyanoacrylate) selected very early rebleeding as the strongest independent predictor for in-hospital mortality $(p<0.001)$.

Follow up During the follow up period (median 19 months, range $0.5-126$ months) $35 \%$ of the patients presented new episodes of GI bleeding, mostly from esophageal varices. Reinjection with cyanoacrylate for poor gastric variceal obliteration or contiguous unobliterated gastric varices detected in a follow up session was performed in 12 $(12.4 \%)$ patients. Overall mortality rate was $58.8 \%$, resulting in most of the cases from hepatic failure deterioration. It was associated with class $\mathrm{C}$ of Child-Pugh classification in cirrhotic patients $(p=0.05)$.

Complications Complications of the procedure occurred in 17 patients $(17.5 \%)$, including pyrexia $\left(>38^{\circ} \mathrm{C}\right)$ in $14.4 \%$, pulmonary embolism in $5.2 \%$ and cerebral embolism in $3.1 \%$. The factors associated with complications were the need for Sengstaken-Blakemore tube before cyanoacrylate and very early rebleeding $(p<0.05)$.

\section{Discussion}

Approximately $20 \%$ to $30 \%$ of cirrhotic patients will experience variceal bleeding within 2 years after diagnosis. In $10 \%$ to $20 \%$ of the cases the source is from gastric varices, with a higher bleeding incidence for fundal varices. Contrasting to esophageal varices, gastric varices represent a particular therapeutic challenge because of their size and location, more severe course, and worse outcomes. In addition to the endoscopic criteria (size of the largest varix and presence of red-spots), poor hepatic function is the most important factor predicting hemorrhage from gastric varices [15]. Because of its excellent efficacy, N-butyl-2-cyanoacrylate is considered to be the optimal endoscopic therapy for gastric variceal bleeding [14]. Indeed, a large number of case series have demonstrated that N-butyl-2-cyanoacrylate injection successfully controlled acute gastric varices, with overall success rates ranging between $70 \%$ to $100 \%$ [3-10]. Radiologic transjugular intrahepatic portosystemic shunt (TIPS) placement has also been used as a therapeutic modality in this setting. However, endoscopic cyanoacrylate is safe and as clinically effective as TIPS, with a lower rate of long-term morbidity and much lower cost [16].

Despite the extensive worldwide use of cyanoacrylate, there are still differences related to the technique, safety and long-term results. Our study was conducted to address these issues of safety and efficacy. Ninety-seven patients with bleeding gastric varices were treated endoscopically with intravariceal injection of N-butyl-2-cyanoacrylate. At the time of endoscopy most of patients 77 (79.4 \%) had only stigmata of recent variceal bleeding. This may be due to the effect of vasoactive drugs (octreotide or terlipressin) given to all patients before endoscopy and of the use of temporary Sengstaken-Blakemore tube before cyanoacrylate.

Hemostatic control was successful in $95.9 \%$ of the patients, comparable to or better than that in other series. The excellent results in primary hemostasis with cyanoacrylate are the consequence of the immediate occlusion of the lumen of the varix, pointing out to the need to inject the mixture near the rupture point. The primary hemostasis was independent of the type of gastric varices and severity of hepatic dysfunction. The overall early rebleeding rate was $14.4 \%$, of which $3.1 \%$ was classified as very early rebleeding and $11.3 \%$ as early rebleeding. Early rebleeding rate after endoscopic treatment with cyanoacrylate has been reported to vary between $0 \%$ to $23 \%$, suggesting that although acute hemostasis is effective, the risk of rebleeding should not be neglected. Complete obliteration of the varices during the initial session or follow up reinjection sessions is essential to achieve complete variceal eradication and prevent rebleeding.

The rebleeding rate was independent of the type of varix. It was $19 \%$ in GOV1, $11 \%$ in GOV2, $13 \%$ in IGV1 and $0 \%$ in IGV2. Fundal varices, like GOV2 and IGV1 of Sarin classification, are known to be associated with a high-flow vessel originating in gastro-renal, gastrophrenic or cardiophrenic shunts, and this distinctive anatomy and physiology contributes to a more aggressive bleeding potential [17]. Kind et al. reported similar rebleeding rates among GOV1 $(19.6 \%)$ and GOV2 (18.9\%) varices but higher rebleeding rate in IGV1 (66.5\%) [18]. However, in our series, that was not verified. Kim et al. also found that a size of the varix greater than $5 \mathrm{~mm}$ and an advanced Child-Pugh class were associated with a high risk of bleeding [15].

Endoscopic related complications from cyanoacrylate injection are typically minor (fever and abdominal discomfort). However, major fatal complications, usually associated with glue embolism have been described [5, 19]. In our patients, one or more complications related to cyanoacrylate injection were seen in 17 patients $(17.5 \%)$, most of them with pyrexia (14.4\%) but also including cases of pulmonary embolism (5.2\%) and cerebral embolism (3.1\%). The occurrence of systemic embolization with cyanoacrylate injection has been associated with a large volume of injection, to the speed of injection and to the existence of shunts between portal and pulmonary system, which are common in cirrhotic patients [19]. In our study, there were no significant differences in the total volume of injection between patients with or without embolic complications $(1.9$ vs. $1.7 \mathrm{~mL})$. The factors associated with occurrence of complications $(p<0.05)$ were the need for Sengstaken-Blakemore tube before cyanoacrylate and very 
early rebleeding. In fact, Sengstaken-Blakemore tube in the management of acute variceal hemorrhage has been associated with a greater risk of complications, namely aspiration pneumonia [20].

In-hospital mortality rate was $9.3 \%$, which is consistent with the reported mortality in other studies. Kind et al. reported that hospital mortality rate was $19.5 \%$, and it was associated to liver failure ( $76 \%$ of cases) and hemorrhagic shock $(8.8 \%)$ [18]. In our study, factors that were associated with in-hospital mortality, were: poor hepatic function (Child-Pugh C), no immediate hemostasis with cyanoacrylate, need for Sengstaken-Blakemore tube after cyanoacrylate, presence of complications, very early rebleeding and prothrombin rate $<42 \%$. Of all these factors, very early rebleeding (occurring within 48 to $120 \mathrm{~h}$ of initial hemostasis) was the strongest independent predictor for inhospital mortality. In fact, mortality reached $28.6 \%$ in the 7 patients with persistent bleeding or with very early rebleeding. The treatment failure-related mortality rate was $2 \%(2$ of 97). Huang et al. also verify that the mortality due to rebleeding immediately after cyanoacrylate sclerotherapy was $12.5 \%$ and treatment failure-related mortality rate was $2.2 \%[5]$.

Overall mortality rate was $58.8 \%$, comparable to other series previously reported $[3,5]$. Similar to these series, where the determining factor for long term survival was the underlying disease leading to portal hypertension, mortality was associated with the degree of the hepatic dysfunction, namely Child-Pugh class C $(p<0.05)$.

In conclusion, N-butyl-2-cyanoacrylate injection is a rapid, low-cost and simple therapeutic option for acute gastric variceal bleeding. It is highly effective for achieving immediate hemostasis with an acceptable rebleeding rate. As very early rebleeding predicts in-hospital mortality, complete variceal obliteration is essential to achieve good outcomes. Major complications after cyanoacrylate occur but are rare. Taking in account all these results, N-butyl-2-cyanoacrylate injection should be the first choice for bleeding gastric varices.

Disclosure of Interest None declared

\section{References}

1. Carbonell N, Pauwels A, Serfaty L, Fourdan O, Lévy VG, Poupon R. Improved survival after variceal bleeding in patients with cirrhosis over the past two decades. Hepatology. 2004;40:652-9.

2. Soehendra N, Nam VC, Grimm H, Kempeneers I. Endoscopic obliteration of large esophagogastric varices with bucrylate. Endoscopy. 1986;18:25-6.
3. Akahoshi T, Hashizume M, Shimabukuro R, et al. Long-term results of endoscopic Histoacryl injection sclerotherapy for gastric variceal bleeding: a 10-year experience. Surgery. 2002;131:S17681.

4. Mumtaz K, Majid S, Shah H, et al. Prevalence of gastric varices and results of sclerotherapy with N-butyl 2 cyanoacrylate for controlling acute gastric variceal bleeding. World J Gastroenterol. 2007;13:1247-51.

5. Huang YH, Yeh HZ, Chen GH, et al. Endoscopic treatment of bleeding gastric varices by N-butyl-2-cyanoacrylate (Histoacryl) injection: long-term efficacy and safety. Gastrointest Endosc. 2000;52:160-7.

6. Wang YM, Cheng LF, Li N, Wu K, Zhai JS, Wang YW. Study of glue extrusion after endoscopic N-butyl-2-cyanoacrylate injection on gastric variceal bleeding. World J Gastroenterol. 2009;15:494551.

7. Seewald S, Ang TL, Imazu H, et al. A standardized injection technique and regimen ensures success and safety of N-butyl-2cyanoacrylate injection for the treatment of gastric fundal varices. Gastrointest Endosc. 2008;68:447-54.

8. Fry LC, Neumann H, Olano C, Malfertheiner P, Mönkemüller K. Efficacy, complications and clinical outcomes of endoscopic sclerotherapy with N-butyl-2-cyanoacrylate for bleeding gastric varices. Dig Dis. 2008;26:300-3.

9. Marques P, Maluf-Filho F, Kumar A, Matuguma SE, Sakai P, Ishioka S. Long-term outcomes of acute gastric variceal bleeding in 48 patients following treatment with cyanoacrylate. Dig Dis Sci. 2008;53:544-50.

10. Sarin SK. Long-term follow-up of gastric variceal sclerotherapy: an eleven-year experience. Gastrointest Endosc. 1997;46:8-14.

11. Sarin SK, Jain AK, Jain M, Gupta R. A randomized controlled trial of cyanoacrylate versus alcohol injection in patients with isolated fundic varices. Am J Gastroenterol. 2002;97:1010-5.

12. Lo GH, Lai KH, Cheng JS, Chen MH, Chiang HT. A prospective, randomized trial of butyl cyanoacrylate injection versus band ligation in the management of bleeding gastric varices. Hepatology. 2001;33:1060-4.

13. Ramesh J, Limdi JK, Sharma V, Makin AJ. The use of thrombin injections in the management of bleeding gastric varices: a single-center experience. Gastrointest Endosc. 2008;68:877-82.

14. de Franchis R, Baveno V. Faculty. Revising consensus in portal hypertension: report of the Baveno $\mathrm{V}$ consensus workshop on methodology of diagnosis and therapy in portal hypertension. J Hepatol. 2010;53:762-8.

15. Kim T, Shijo H, Kokawa H, et al. Risk factors for hemorrhage from gastric fundal varices. Hepatology. 1997;25:307-12.

16. Procaccini NJ, Al-Osaimi AM, Northup P, Argo C, Caldwell $\mathrm{SH}$. Endoscopic cyanoacrylate versus transjugular intrahepatic portosystemic shunt for gastric variceal bleeding: a singlecenter U.S. analysis. Gastrointest Endosc. 2009;70:881-7.

17. Matsumoto A, Izumiya T, Takimoto K, Inokuchi H. Management of acute gastric variceal bleeding. Aliment Pharmacol Ther. 2003; $18: 1173-4$.

18. Kind R, Guglielmi A, Rodella L, et al. Bucrylate treatment of bleeding gastric varices: 12 years' experience. Endoscopy. 2000;32:512-9.

19. Soehendra N, Grimm H, Nam VC, Berger B. N-butyl-2-cyanoacrylate: a supplement to endoscopic sclerotherapy. Endoscopy. 1987;19:221-4.

20. Lo GH, Lai KH, Ng WW, et al. Injection sclerotherapy preceded by esophageal tamponade versus immediate sclerotherapy in arresting active variceal bleeding: a prospective randomized trial. Gastrointest Endosc. 1992;38:421-4. 